\title{
Series televisivas como espacios de negociación de la feminidad, la masculinidad y las expectativas sociales en el Japón contemporáneo
}

\author{
Television series as spaces for negotiation \\ of femininity, masculinity, and social expectations \\ in contemporary Japan
}

DOI: 10.32870/mycp.v10i28.711

\begin{abstract}
Resumen
En este artículo se analizan los discursos hegemónicos sobre la feminidad y masculinidad, las expectativas sociales y la valoración que se hace de los individuos de acuerdo con su estado civil, centrando la atención en las presiones sociales a las que son expuestas las personas solteras y sin hijos en el Japón contemporáneo. Desde la perspectiva de los estudios culturales, se consideran los discursos sociales que relacionan la feminidad y la masculinidad con el matrimonio y la maternidad/paternidad, y se realiza un análisis textual interpretativo de una muestra de series televisivas para discutir las ideas dominantes que circulan en la sociedad japonesa acerca del creciente número de personas solteras. Se argumenta la posibilidad de que ellas estén encontrando en los contenidos televisivos un espacio de negociación con la sociedad para defender sus trayectorias de vida y rechazar las etiquetas que se les han estado imponiendo.
\end{abstract}

Palabras clave: Japón, feminidad, masculinidad, discursos hegemónicos, representación en televisión.
Yunuen Ysela Mandujano-Salazar ${ }^{1}$

\begin{abstract}
This article analyzes the hegemonic discourses about femininity and masculinity, social expectations, and social assessment of individuals according to their marital status, focusing the attention on the social pressure put upon single and childfree individuals in contemporary Japan. From the perspective of the cultural studies, considering the public social discourses that relate femininity and masculinity with marriage and motherhood/ fatherhood, and the interpretative textual analysis of a sample of television series, the dominant ideas circulating in Japan about these people are discussed. It is argued the possibility of them finding in these television contents a platform to negotiate with society and defend their life trajectories and to reject the labels that have been imposed on them.
\end{abstract}

Keywords: Japan, femininity, masculinity, hegemonic discourses, televisual representation.

Artículo recibido el 18 de julio de 2020 y dictaminado el 16 de septiembre de 2020.

1. Universidad Autónoma de Ciudad Juárez. Av. Plutarco Elías Calles 1210 Fovissste Chamizal, Cd. Juárez, Chihuahua, México, C.P. 32310. ORCID: 0000-0003-4794-6584. Correo electrónico: yunuen.mandujano@uacj.mx 


\section{Introducción}

En la década de 1980, en medio de la burbuja económica y con la paulatina erosión del ideal de la clase media nacional, surgieron las series televisivas de ficción conocidas como trendy dramas, convirtiéndose en uno de los principales productos mediáticos dentro de Japón. Tal como dice Lukács (2010), la característica principal de este tipo de contenidos ha sido representar estilos de vida vigentes en la sociedad japonesa al momento de su producción y ser un catálogo de los múltiples productos y servicios destinados a explotar los deseos y el ingreso disponible de la audiencia a la que van dirigidos. Lukács señala que en este tipo de producciones no se explora en profundidad el contexto social como elemento de construcción del individuo, pues la trama se crea alrededor de los personajes protagónicos y de algún tema específico. No obstante, aquí lo que se busca comprobar es que, a partir del siglo XXI y conforme la audiencia principal de los trendy dramas - las mujeres solteras-se ha vuelto objeto de debate político y social debido a la problemática demográfica que enfrenta Japón; estos contenidos se han convertido en espacios de negociación ideológica entre las personas solteras y otros actores sociales $\mathrm{y}$, por tanto, constituyen importantes muestras de las expectativas y crisis de la sociedad japonesa contemporánea.

En este sentido, desde el cambio de milenio, una tendencia respecto a los personajes protagónicos de los trendy dramas se ha hecho evidente: cada vez son más las historias centradas en personajes mayores de 30 años - principalmente mujeres, pero también algunos varones - renuentes a alcanzar los marcadores normativos de adultez del matrimonio y la maternidad/paternidad (Dales, 2014; Mandujano-Salazar, 2017). Más relevante es que, en el periodo de 2001 a 2019, 13 dramas de horario estelar fueron producidos por alguna de las tres mayores televisoras privadas del país, enfocándose en personajes que rebasaban los 30 años siendo solteros y, luego de pasar por las presiones sociales debido a su estado civil y su edad, al final de la serie reafirman su decisión de permanecer solteros y sin hijos.

Esto indica la relevancia que este sector de la población ha adquirido en la sociedad japonesa contemporánea. Conforme la soltería entre las personas de más de 30 años se ha incrementado en las últimas décadas, éstas han sido acusadas de ser las principales culpables detrás de la crisis demográfica de la nación. Su desviación del curso de vida socialmente preferido se ha vuelto un tema recurrente en la televisión nipona, lo cual ha apoyado el uso público 
de etiquetas para categorizarlas, manifiesta o sutilmente, como perdedoras, particularmente a las mujeres (Mandujano-Salazar, 2017).

En el presente artículo, escrito desde la perspectiva de los estudios culturales y desde un análisis textual interpretativo, el objetivo es identificar cuáles han sido los discursos dominantes que circulan en la sociedad japonesa de las primeras décadas del siglo XXI y que son reflejados en los trendy dramas de ese país acerca de las personas solteras y sin hijos de más de 30 años, en medio de la coyuntura demográfica de las últimas décadas. Se considera que este tipo de contenidos mediáticos son una muestra de la evolución de las luchas ideológicas que están aconteciendo en la sociedad japonesa y se argumenta la posibilidad de que las personas solteras hayan comenzado a reclamar el control sobre cómo definir su feminidad, masculinidad, trayectorias de vida, éxito y felicidad.

\section{Metodología}

La interpretación de textos mediáticos es compleja y requiere varios niveles de análisis. Primeramente, es esencial ubicar claramente tanto el contexto sociocultural de producción como el de recepción de interés. Un mismo texto tendrá interpretaciones potencialmente distintas dependiendo del contexto en el que se localiza la audiencia y su exposición a otros textos y metatextos.

Dado el interés ya establecido en este artículo, es fundamental ofrecer un análisis contextual del modelo hegemónico de feminidad y masculinidad en el Japón de la posguerra y de cómo, para finales del siglo Xx, los cambios en las condiciones demográficas convirtieron a las personas adultas solteras y sin hijos en un tema recurrente en la sociedad. Esto servirá de metatexto para la interpretación, pues identificará aquellos discursos dominantes con los que crecieron las generaciones de japoneses que en las primeras décadas del siglo XXI se encuentran en medio del debate de interés.

Luego, se presentarán las principales nociones que se convirtieron en etiquetas de uso público y mediático para referirse a este tipo de personas y que reflejaban la ansiedad social y gubernamental hacia ellos. Analizar su surgimiento y uso en el discurso público servirá como parte del análisis intertextual.

Ahora bien, los trendy dramas son representaciones de un género definido que ya por sí mismo contiene significados. Por tanto, en otra sección se dará 
un panorama del tipo de series que se están analizando y su relevancia en el entretenimiento mediático del Japón contemporáneo.

Finalmente, para el análisis textual se eligió una muestra de cuatro trendy dramas basada en los siguientes criterios: distribución temporal en una década (2006-2016), relevancia discursiva y ratings en televisión abierta japonesa durante su transmisión por primera vez. En este sentido, es importante señalar que, con la alta segmentación de mercado y la aparición de nuevas tecnologías de comunicación y difusión de contenidos, los ratings de la televisión japonesa han ido cayendo consistentemente. Entre el año 2000 y 2009, un trendy drama que alcanzara arriba de 10 puntos era considerado un éxito. Sin embargo, desde la segunda década del siglo se comenzó a popularizar el servicio de video streaming a través de proveedores como Hulu, Netflix, Amazon Prime Video, entre otros, por lo que los ratings promedio han ido cayendo más. Esto, no obstante, no significa que los trendy dramas producidos y transmitidos en principio por las cadenas de televisión abierta hayan perdido relevancia social, puesto que éstas se han encargado de llevar sus contenidos a las opciones streaming, ya sea por medio de sus propios sitios o vendiendo derechos de distribución a los proveedores antes mencionados. Por tanto, ahora los contenidos tienen un alcance mayor, lo que ha ido perdiendo significado en los últimos años son los ratings tradicionales.

Bajo las consideraciones anteriores, se seleccionaron las siguientes series para la muestra: Kekkon dekinai otoko ("El hombre que no se puede casar"), transmitida por primera vez en el verano de 2006, alcanzando un rating promedio de 16.9 puntos; Around 40 ("Alrededor de 40"), transmitida en la primavera de 2008 con rating promedio de 14.6; Kekkon shinai, Wonderful single life ("No me caso, maravillosa vida de soltería"), transmitida originalmente en el otoño de 2012, con rating promedio de 11.8; y Watashi kekkon dekinainjanakute, shinaindesu ("No es que no me pueda casar, es que no me caso"), transmitida en la primavera de 2016, con rating promedio de 8.99 (Video Research Ltd, s.f.).

En el análisis de estas series se consideraron la representación visual, los gestos y la comunicación verbal entre personajes como elementos que se interpretaron en el contexto sociocultural en el que se dio la producción y transmisión originales. Asimismo, las narrativas de las historias se discuten triangulándolas con el metatexto y los otros textos relacionados - las etiquetas a la soltería y las otras series del mismo género- a fin de develar los discursos que envuelven acerca de la feminidad, la masculinidad, las ex- 
pectativas sociales y la valoración hacia mujeres y varones de acuerdo con su falta de cumplimiento de los papeles de esposos y madre/padre.

\section{Los modelos hegemónicos de feminidad y masculinidad del Japón próspero y la crisis demográfica del siglo XXI}

Durante las últimas décadas del siglo XIX apareció el lema de "buenas esposas, madres sabias" (ryōsai kenbo) y, desde entonces, durante gran parte del siglo $\mathrm{xx}$ esta ideología continuó siendo activamente promovida por el Gobierno a través de la educación, volviendo hegemónico el discurso que dictaba que el papel principal de la mujer era el de ser esposa y madre y su deber nacional era el educar a sus hijos (Fujimura-Fanselow, 1991; Niwa \& Yoda, 1993). Por su parte, los varones debían servir a su nación apoyando directamente en su progreso económico o defendiendo sus intereses a través de los papeles de empresarios y trabajadores - o soldados, en la época de las guerras (Dasgupta, 2010; Hidaka, 2010).

Luego del fin de la Guerra del Pacífico, el orden democrático impuesto a Japón por las fuerzas de ocupación, lideradas por Estados Unidos, afectó a la sociedad en muchos aspectos, entre ellos el estatus legal igualitario dado a hombres y mujeres. En ese momento, las mujeres japonesas obtuvieron el derecho a poseer y manejar propiedades y a tener —en teoría - las mismas oportunidades laborales y educativas que los varones (Buckley, 1993). Asimismo, el contacto con ideas occidentales despertó la conciencia feminista en algunas mujeres, quienes trataron de retar las ideas patriarcales dominantes que dictaban que la maternidad era un aspecto esencial de la mujer (McKinlay, 2002).

A pesar de ello, en las décadas de rápida industrialización y aceleración económica de posguerra, las élites japonesas - Gobierno, corporaciones, líderes intelectuales, medios - promovieron activamente un modelo de familia nuclear perteneciente a una llamada nueva clase media que se decía representativa del éxito social en esa nueva etapa de prosperidad (Vogel, 1971). Este modelo de familia ideal estaba encabezado por el salaryman - empleado de cuello blanco de las grandes corporaciones y las oficinas gubernamentales- y la sengyōshufu —ama de casa de tiempo completo—, quienes se convertirían en arquetipos de masculinidad y feminidad y que estaban intrínsecamente relacionados con el matrimonio heterosexual, la reproducción y la división del trabajo dentro y fuera del hogar. Estos modelos dicótomos de género se volvieron parte del 
discurso hegemónico de identidad nacional del milagro económico — conocido como nihonron o nihonjinron -, el cual promovía los papeles y deberes civiles de acuerdo con una ideología patriarcal basada en algunas ideas de décadas anteriores pero adecuada a las nuevas metas nacionales: el varón como trabajador que dominaba la esfera pública; la mujer como esposa y madre que se mantenía en la esfera privada (Mandujano-Salazar, 2017).

Casi como heredero del samurai de la época feudal y del soldado de los tiempos de guerras, el salaryman personificaba la principal figura masculina del Japón industrializado: un hombre entregado a su trabajo que participaba en el objetivo nacional de convertir a la nación en una potencia económica a nivel mundial (Dasgupta, 2010; Hidaka, 2010; Mandujano-Salazar, 2017). Debido a las exigencias laborales a las que era expuesto el salaryman, era mínimo el tiempo que podía pasar de convivencia con su familia, aunque su función reproductiva era parte esencial del modelo social. Así que, al casarse, era esperado que su esposa se convirtiera en sengyōshufu para asumir la responsabilidad de todas las labores domésticas y, más importante, del cuidado y educación de los hijos.

Durante estas décadas de crecimiento económico sostenido, la televisión se convirtió en el medio de comunicación masiva más poderoso del país, moldeando no sólo los gustos de consumo de la gente, sino también diversas expresiones culturales e ideales sociales (Ivy, 1993). Apoyados por la televisión, los discursos sobre la responsabilidad de las mujeres en la crianza de los hijos y la administración del hogar fueron constantes; en los contenidos televisivos se promovían prácticas e imágenes que implícitamente dictaban que el mejor lugar para ellas era en el hogar, teniendo hijos y educándolos (Uno, 1993). Al mismo tiempo, las series televisivas de la época representaban hogares de clase media centrados en la figura de la sengyōshufu y evocaban éste como el estilo de vida deseable y más gratificante para la mujer al simbolizar su éxito social de conseguir un marido con suficiente estabilidad laboral y económica para que ella pudiera dedicarse a las labores del hogar y la crianza de la siguiente generación de ciudadanos (Luckács, 2010; Mandujano-Salazar, 2017).

Además, el Gobierno y la industria, siguiendo intrínsecamente la ideología de género hegemónica, le negaban sistemáticamente a las mujeres las mismas oportunidades de progreso laboral que a los varones, pues no les ofrecían contratos permanentes ni los mismos salarios. Las mujeres solían ser empleadas en roles de apoyo y no especializados, generalmente prefiriendo a las jóvenes solteras, pues la expectativa era que, cuando se casaran, se re- 
tirarían y dejarían su puesto a otras más jóvenes (Yu, 2009). Así, la sociedad japonesa de posguerra y del milagro económico valoraba y celebraba el modelo de "buenas esposas, madres sabias" de tiempo completo que complementaba el del empleado varón de tiempo completo. El matrimonio y la maternidad se ponían como base para el desenvolvimiento de la feminidad japonesa socialmente deseable, mientras que la masculinidad se consideraba reflejada en la capacidad y entrega del hombre en su trabajo y el cumplimiento de ser sostén económico de su familia.

Estos modelos hegemónicos de género pudieron sostenerse unas décadas por el vigor de la economía de posguerra, que pudo prescindir hasta cierto grado de las mujeres en la fuerza laboral. Las curvas de fuerza laboral femenina en pronunciada forma de "M" se volvieron representativas de las décadas de 1960 a 1980, reflejando que aproximadamente la mitad de la población femenina seguía el curso de vida socialmente deseado: un alto porcentaje de mujeres jóvenes entraba a la fuerza laboral entre el fin de su educación media superior y antes de casarse; durante la primera mitad de los veinte, muchas se retiraban para convertirse en sengyōshufu; y, luego de que sus hijos entraban a la escuela, algunas de esas que se habían retirado volvían al trabajo, aunque mayormente en puestos de tiempo parcial (Buckley, 1993; Upham, 1993).

No obstante, conforme se acercaba el cambio de siglo, hombres y mujeres fueron retardando cada vez más el momento del matrimonio. La edad media de primer matrimonio para los varones pasó de 26.9 años en 1970 a 28.8 en 2000; en las mujeres pasó de 24.2 a 27.0 en el mismo periodo (National Institute of Population and Social Security Research, s.f.). Esto se puede relacionar con dos factores principales: su ingreso a instituciones de educación superior y su participación en la fuerza laboral (Mandujano-Salazar, 2017). Conforme las mujeres comenzaron a retrasar el matrimonio, la tasa de fecundidad en Japón comenzó a caer por debajo de la tasa de reemplazo; desde 1974, la tasa ha sido de menos de 2.1, disminuyendo paulatinamente hasta alcanzar su punto más bajo en 2005 con 1.26 (National Institute of Population and Social Security Research, 2017). La reacción por parte de los líderes ideológicos, del Gobierno y la industria no se hizo esperar. En una sociedad en donde la reproducción se ha mantenido altamente relacionada con el matrimonio (Ishida, 2013; Rosenberger, 2007), pronto aparecieron los debates en donde se culpaba principalmente a las mujeres y su mayor presencia en las universidades y los centros de trabajo (Buckley, 1993). 
A pesar del pánico entre las élites por la aparente rebelión femenina, las estadísticas muestran que, todavía en 1980, la proporción de mujeres nunca casadas de entre 25 y 29 años era menos del 25\% y la participación laboral para el mismo grupo decrecía significativamente en comparación con el grupo de edad más joven (Mandujano-Salazar, 2017). Esto significa que, hasta ese momento, convertirse en sengyōshufu continuaba siendo la elección de la mayoría de las mujeres; sólo esperaban unos años más para casarse y tenían menos hijos que las generaciones anteriores. Fue después de la explosión de la economía de la burbuja y la subsecuente crisis económica en la década de 1990, cuando la viabilidad del hogar centrado en la sengyōshufu y la fuerza laboral centrada en el salaryman comenzaron a decaer, acelerando cambios en la forma en que los jóvenes japoneses, particularmente las mujeres, elegían sus trayectorias de vida.

Los censos de población recientes han mostrado una creciente tendencia a la soltería entre las mujeres de los grupos de edad que se consideran como los más fértiles. En 2015, sólo el $8 \%$ de las mujeres de entre 20 y 24 años y el $36.3 \%$ de las de 25 a 29 estaban casadas; por otro lado, el $34.6 \%$ de las mujeres de 30 a 34 años y el $23.9 \%$ de las de 35 a 39 nunca se habían casado; en el caso de los varones, permanecían sin casarse el 35.0\% del grupo de 35 a 39 años y el 30.0\% de los de 40 a 44 (National Institute of Population and Social Security Research, 2017). Estas cifras reflejan que la soltería incluso alrededor de los 40 años se ha vuelto más frecuente entre los japoneses.

Entrada la segunda década del siglo XxI, el Gobierno japonés se ha encontrado en una encrucijada. Si bien ha tenido que reconocer la importancia de la participación femenina en la fuerza laboral, también ha tenido que continuar promoviendo la importancia que tiene su papel social como madre. Particularmente desde diciembre de 2012, con la llegada de Shinzō Abe a la silla de primer ministro, las mujeres como trabajadoras y como madres se han convertido en el foco de estrategias gubernamentales que tratan de revitalizar la economía nacional y remediar la crisis demográfica. Abenomics, una serie de reformas estructurales promovidas por Abe, han incluido medidas para impulsar la inclusión de las mujeres en empleos a todos los niveles y para proveerles de un ambiente en el que puedan desarrollarse profesionalmente mientras crían a sus hijos (Prime Minister of Japan and His Cabinet, 2014). Con ese fin, el gobierno de Abe comenzó a trabajar para incrementar los centros de cuidado infantil y la capacidad de los mismos, a promover que más hombres tomen un periodo de descanso laboral por paternidad, así como a 
ofrecer estímulos a las empresas que incrementen la proporción de mujeres en posiciones ejecutivas - políticas apodadas womenomics (The Government of Japan, 2018).

Sin embargo, los resultados aún no son particularmente perceptibles. La industria y la sociedad japonesa han demostrado ser renuentes a cambiar sus visiones acerca de los principales papeles y el lugar de las mujeres, volviéndoles difícil a éstas ser esposas, madres y trabajadoras al mismo tiempo. La resistencia encontrada por las mujeres en sus familias y - potenciales- esposos, así como las dificultades encontradas en los lugares de trabajo, han hecho que numerosas mujeres deban elegir entre tener una profesión o tener una familia. Esto se ve reflejado en múltiples reportajes acerca del acoso y presiones que las madres trabajadoras enfrentan (Cox, 2015; Ryan, 2015). Es evidente que elementos culturales, actitudes sociales y estereotipos acerca de las mujeres y la relación de la feminidad con el matrimonio y la maternidad no han cambiado mayormente para lo que en Japón se llama el seken, un concepto que involucra tanto al público, a la comunidad y a la visión hegemónica y que se impone más allá de los deseos del individuo, constituyéndose en la audiencia y el juez social que rodea a la persona (Lebra, 2004).

\section{Las etiquetas de la soltería en la sociedad japonesa}

La creciente tendencia entre personas japonesas a desviarse de la trayectoria de vida socialmente deseable que implica el pronto matrimonio y la procreación ha sido causa de mucho debate social. Éste se ha visto reflejado en el surgimiento de diversas nociones utilizadas para etiquetar principalmente a las mujeres solteras como un grupo social problemático.

Desde hace décadas los medios japoneses - prensa, radio, televisión, cine- han promovido el uso de ciertos términos con connotaciones peyorativas para referirse a las personas, particularmente a las mujeres, y su valía —o falta de ésta- que, cerca o pasada cierta edad considerada socialmente ideal para el matrimonio, permanecen solteras: desde "pastel de navidad" y "soltero parásito", hasta makeinu - perro perdedor-y ohitorishama — persona sola (Mandujano-Salazar, 2017). Por medio de estas etiquetas los medios de comunicación japoneses han estado dispersando discursos que promueven estereotipos sobre las personas y sus decisiones de vida y que tienen el potencial de impactar cómo las trata la sociedad y cómo se perciben ellas mismas. 
En la década de 1970, la norma social era que las mujeres se casaran antes de los 25 años. Como consecuencia, todavía en la década de 1980 un término era popular en el discurso público para referirse al valor de las mujeres solteras en el mercado de matrimonios: pastel de navidad, una noción peyorativa disfrazada con un tono festivo. Implicaba que las mujeres solteras tenían un elevado valor y estaban en alta demanda antes de cumplir 25 años - tal como los pasteles de navidad eran muy buscados días antes del 25 de diciembrepero que, una vez llegadas a esa edad, su valor decaía rápidamente y ya no eran ni buscadas ni necesitadas (Takemaru, 2010).

A pesar de la presión social, se estaba volviendo obvio que cada vez a menos mujeres les interesaba casarse después de su fecha de expiración de 25 años. Por el contrario, un mayor número de mujeres permanecían solteras por más tiempo para enfocarse en sus carreras y disfrutar su libertad o porque tenían dificultades para encontrar parejas que cumplieran con sus expectativas. Pues, conforme las mujeres se volvían más independientes, ideológica y financieramente, los varones también comenzaron a experimentar la adultez en formas diferentes a las generaciones previas. Los hombres jóvenes comenzaron a tener dificultades para encontrar trabajos permanentes y con sueldos competitivos, algo que influenciaba sus posibilidades de encontrar una mujer que quisiera casarse con ellos. Pero, también, muchos de ellos se estaban volviendo más dispuestos a explorar sus deseos y los límites de sus personalidades, alejándose del modelo de masculinidad hegemónica y rechazando comprometerse en relaciones tradicionales.

Así, para el final de la década de 1990, conforme la crisis económica postburbuja evidenciaba las difíciles tendencias demográficas, las voces conservadoras del país comenzaron a culpar de la situación a los solteros parásitos. Hecha popular por el sociólogo Yamada (1999), esta noción se refería a los solteros y sin hijos - hombres y mujeres - que vivían en la casa paterna luego de terminar sus estudios universitarios. Él los consideraba egocéntricos, incluso antipatrióticos por no contribuir con la sociedad. Diversas opiniones aparecieron alrededor de sus postulados, pero el término se volvió ampliamente utilizado por los medios para esparcir el tono acusador contra la gente soltera y sus estilos de vida; en particular, a través de esta noción se generalizó la imagen de las mujeres solteras adultas como frívolas y superficiales.

A inicios del siglo xxI, Ohitorisama (Iwashita, 2001), un libro dedicado a mostrarles a las mujeres adultas cómo disfrutar la vida sin necesidad de compañía — viajando, saliendo a comer, bebiendo, etc. - fue publicado. La 
autora estaba tratando sólo el tema de cómo las mujeres podían disfrutar solas de actividades que eran usualmente consideradas para parejas o grupos. Ella no discutía particularmente el estado civil de sus lectoras, sólo la actitud independiente y audaz, y el estilo de vida surgido de una razonable capacidad económica, que estas mujeres tenían en común. Fue entonces que el término ohitorisama - literalmente "una persona" - comenzó a ser utilizado con la connotación de "mujeres independientes".

Mientras estas mujeres poco ortodoxas continuaban en el centro del debate social, la noción de makeinu - literalmente "perro perdedor"- se volvió popular gracias a otro libro, Makeinu no tōboe (Sakai, 2003), escrito por una ensayista de 37 años de edad, soltera y sin hijos; el libro trataba de recuperar el orgullo de las mujeres solteras, invitándolas a aceptar que, de acuerdo con el seken, la máxima meta en la vida de una mujer era ser esposa y madre, por lo cual aquellas que no lo eran serían percibidas como perdedoras en el contexto social. La autora defendía que, a pesar de ello, las mujeres señaladas socialmente como makeinu tenían razones para sentirse orgullosas: típicamente, eran educadas, valientes y económicamente independientes. Sin embargo, en el uso televisivo el término se volvió mayormente usado como una burla hacia las mujeres de más de 30 años que permanecían solteras y sin hijos, dejando de lado la intención de la autora e imponiendo la connotación negativa a la etiqueta (Mandujano-Salazar, 2017).

Durante la segunda mitad de la década de 2000, conforme la noción de makeinu circulaba en las narrativas mediáticas de entretenimiento, ohitorisama estaba siendo utilizada por diferentes industrias de productos y servicios para explotar el segmento de mercado de personas solteras - principalmente mujeres - que se identificaban a sí mismas con el modelo de solteros independientes. Luego, algunos escritores y académicos comenzaron a adoptar también el término como una alternativa menos despectiva para discutir y referirse particularmente a las mujeres que, teniendo más de 30 años, seguían solteras y sin hijos (Dales, 2014). Los medios siguieron la tendencia y comenzaron a sustituir la noción de makeinu por ohitorisama en la mayoría de sus contenidos.

Aunque las etiquetas han cambiado, el juicio hacia las personas solteras que no se encuentran dentro de la norma social ha permanecido, aunque hacia los varones no se ha plasmado en etiquetas tan peyorativas. Surgieron términos para etiquetar a las personas, pero en este caso no de acuerdo con su estado civil, sino con su rango de edad, se volvió de uso muy común: arafō. Esta 
palabra comenzó a ser utilizada durante la segunda mitad de la década de los 2000, para referirse a quienes tenían "alrededor de 40 años" —específicamente entre 35 y 44 - Pronto fue evidente que cada vez más personas - hombres y mujeres - arafō podían ser categorizadas también como ohitorisama.

En ese contexto, los trendy dramas abrieron un espacio para presentar historias centradas en personas de más de 30 años, dedicadas a sus carreras, económicamente independientes, solteras y sin hijos, quienes, sin embargo, vivían bajo las presiones familiares, laborales y sociales por no entrar en el molde de familia socialmente deseable.

\section{La dramatización de la soltería}

Desde el cambio de siglo el tema de la gente soltera y sin hijos de más de 30 años se volvió popular en los medios japoneses. Por un lado, el creciente número de hombres y mujeres con estas características creó una audiencia que, para las corporaciones mediáticas, volvía redituable la producción de contenidos dedicados a y enfocados en ellos. Por otro, tales contenidos surgían también como inevitables escaparates para la contienda ideológica entre diferentes actores sociales.

Los trendy dramas, al ser contenidos característicamente coyunturales, rápidamente comenzaron a reflejar las tendencias sociodemográficas y a centrar cada vez más producciones en personajes que, una década antes, era inimaginable considerar como protagónicos: hombres y mujeres que, pasados los 30 años, estaban solteros y no querían o no sabían cómo conseguir pareja, privilegiando su desarrollo profesional y que, al finalizar la historia, a veces encuentran una relación sentimental, pero permanecen sin casarse y sin renunciar a sus carreras, creando sus propios estilos de vida y relaciones sin seguir los parámetros sociales.

Como muestra, basta analizar a las tres mayores televisoras privadas con cobertura abierta nacional: Nippon Television Network (NTV), Fuji Television (Fuji TV) y Tokyo Broadcasting System Television (TBS) que han asignado este tipo de historias a algunos de sus horarios estelares - miércoles, jueves o viernes entre las 21:00 y 23:00 horas- en formatos de ocho a 12 episodios semanales, cada uno con una duración aproximada de 50 minutos. Entre 1991 y 2000, sólo un drama con personajes protagónicos de las características mencionadas fue producido y transmitido: Dokushin seikatsu (TBS, 1999). Entre 2001 y 2010 aumentaron a cuatro: Anego (NTV, 2005), Kekkon dekinai 
otoko (Fuji TV, 2006), Around 40 (тBS, 2008), у Ohitorisama (TBS, 2009). ${ }^{2}$ Para el periodo de 2011 a 2019 la cifra llegó a nueve en horario estelar y uno en horario de sábados a las 23:00 horas: Kekkon shinai, Wonderful single life (Fuji TV, 2012), Dokushin kisoku (Fuji TV, 2013), Last cinderella (Fuji TV, 2013), Otona joshi (Fuji TV, 2015), Gisō no füfu (NTV, 2015), Hayako sensei, kekkon surutte hontō desuka? (Fuji, 2016), Watashi kekkon dekinainjanakute, shinaindesu (TBS, 2016), Tokyo Tarareba Musume (NTv, 2017), Kekkon Aite wa Chusen de (Fuji, 2018), Mada Kekkon Dekinai Otoko (Fuji, 2019).

Es indudable que esta creciente oferta de series centradas en este tipo de personajes es un reflejo de la relevancia económica que están adquiriendo las personas solteras en el Japón contemporáneo. Además, los trendy dramas no dejan de ser productos culturales que responden a una lógica de mercado, pero también tratan de ser dispersoras de discursos hegemónicos, por lo que las series centradas en personajes que representan a esos sectores que se alejan del ideal social se convierten en terrenos de contienda ideológica entre las élites productoras y económicas, y las personas que se ven representadas. Por ello, analizar las representaciones y narrativas de algunas de estas series permite develar la evolución de esas ideologías.

En la siguiente sección se presentarán los resultados del análisis textual interpretativo de la muestra de cuatro series respecto a los tropos que construyen de las mujeres y los hombres solteros sin hijos, de más de 30 años.

\section{Reivindicando la soltería como una forma de vida}

Kekkon dekinai otoko y Around 40 son ejemplos relevantes de los discursos de la primera generación de dramas centrados en la soltería como estilo de vida, los conflictos personales y las presiones sociales a las que se ven sujetas las personas que se deciden por ella. Estas dos series, transmitidas con dos años de diferencia, fueron bastante populares, alcanzando ratings promedio de 16.9 y 14.6 puntos, respectivamente (Video Research Ltd, s.f.). La primera tuvo por guionista a Ozaki Masaya — un hombre de 46 años al momento de la producción-y fue producido por tres varones; en el segundo fue Hashibe Atsuko la guionista, quien tenía 42 años cuando se transmitió el drama, y tuvo a un productor $y$ una productora.

2. Además, en enero de 2005 Makeinu no tōboe fue transmitido por NTV, pero en el formato de drama especial en un solo episodio de dos horas. 
Ambas historias se centraron en personajes que encajaban con las etiquetas tanto de arafō como de ohitorisama - Kuwano Shinsuke (interpretado por Abe Hiroshi) y Ogata Satoko (interpretada por Amami Yuki)—, ambos a punto de cumplir 40 años al inicio de la historia. Estos personajes son representados como independientes, seguros de sí mismos, solteros y aparentemente no interesados en buscar pareja, además de ser altamente educados y enfocados en sus carreras: uno es arquitecto y tiene su propia firma, otra es psiquiatra y está a cargo de su departamento en el hospital en que trabaja.

Las semejanzas terminan ahí. En el caso de Shinsuke, se le representa como un hombre que disfruta estar solo y es capaz de realizar todas las tareas profesionales y del hogar a la perfección. Él mismo se cocina platillos elaborados, mantiene su departamento y su ropa impecables y disfruta de escuchar música sin interrupciones. Es completamente autosuficiente y exitoso en su trabajo, pero es algo huraño. Sus conocidos lo consideran un hombre raro y asumen que esa rareza es por lo que no se ha casado, pero él es feliz con su estilo de vida al acercarse su cuadragésimo cumpleaños.

En el caso de Satoko, sus amplias aptitudes en la esfera profesional son contrastadas con su inhabilidad en las labores domésticas y su gusto por la comedia de gente mayor; su forma madura de hablar y su estilo reservado y sencillo de vestir, discrepaban con la voz suave y la moda kawaii ("linda") de otros personajes femeninos representados en la historia. Estas características refuerzan su estatus fuera del modelo de feminidad hegemónica. Aunque Satoko no se niega a la posibilidad de casarse, tampoco buscaba activamente pareja, por estar concentrada en su trabajo. No obstante, al acercarse a los 40 años asiste a una reunión con compañeros de escuela y nota que la gente la ve con cierta pena y le hace comentarios de ánimo, como si ella estuviera en una condición de derrota o retraso. Al percibir esta condescendencia, se comienza a cuestionar sobre su soltería. En este caso, el cuestionamiento a su alrededor es sobre su felicidad y logros personales en esa etapa de su vida; su éxito profesional y el hecho de que ella disfrute su vida de soltera parecen ser obviados por el resto de las personas, quienes le imponen su interpretación de que una mujer sin marido ni hijos no puede ser verdaderamente feliz. Eventualmente, Satoko se deja llevar por esta visión externa y comienza a permitir que la gente alrededor intervenga tratando de conseguirle un potencial marido.

Ambas historias vuelven a tener un paralelo en su desarrollo cuando, sin planearlo ni desearlo, desarrollan un interés romántico por alguien que tampoco busca las típicas trayectorias de vida. En el caso de Shinsuke, se ve 
interactuando particularmente con Natsukawa Yui (interpretada por Hayasaka Natsumi), quien es una médico internista reconocida, arafō, y que por años había estado negada a enamorarse. A través de la convivencia, ambos van deconstruyendo sus prejuicios, y al final de la serie deciden intentar llevar una relación sin casarse, sin vivir juntos y sin renunciar a sus estilos de vida y prioridades profesionales.

En el caso de Satoko, se encuentra de pronto conviviendo con un psicólogo de 33 años que es puesto bajo su guía en el hospital, Okamura Keitarō (interpretado por Fujiki Naohito). Este personaje destaca por ser un hombre inexperto en el campo laboral al que llega y por estar claramente fuera del modelo hegemónico de masculinidad japonesa, encajando, en cambio, en el modelo de los sōshoku danshi - chicos herbívoros- que se caracterizan por ser sensibles y pasivos. Esta característica es reforzada simbólicamente por su preocupación por economizar y su preocupación por ayudar a los demás, lo cual se considera una característica de las amas de casa. En un inicio, Satoko se resiste a aceptar que tiene sentimientos hacia él, pues no cumple con sus expectativas de pareja al ser considerablemente más joven, inexperto e inestable económicamente. En un punto de la historia, Satoko es pretendida por un hombre que personifica la masculinidad hegemónica y que le ofrece la posibilidad de alcanzar la meta del matrimonio, pero que no comprende ni acepta por su estilo de vida. Al final de la historia, Satoko y Keitarō inician una relación a distancia, pues a éste se le presenta la oportunidad de desarrollar un proyecto en otra ciudad y a Satoko de quedar a cargo del hospital en que trabaja.

En contraste con los dos dramas anteriores, Kekkon shinai, Wonderful single life y Watashi kekkon dekinainjanakute, shinaindesu dieron paso al involucrar, desde el título, la voz activa de los protagonistas, quienes declaraban estar tomando una decisión sobre sus vidas.

Kekkon shinai, por medio de una narrativa realista creada por las guionistas Yamazaki Takako y Sakaguchi Riko —ellas mismas arafō cuando el drama fue grabado y transmitido originalmente- presentó una historia introspectiva centrada en dos arafō que enfrentaban de diferente forma su soltería: Tanaka Chiharu (interpretada por Kanno Miho) y Kirishima Haruko (interpretada por Amami Yuki, la misma actriz que protagonizó Around 40).

Chiharu, quien cumple 35 años durante el primer episodio, es una empleada temporal en una agencia de viajes y parece vivir al día, sin planear su futuro. Vive con sus padres, no tiene un trabajo permanente y, cuando una 
compañera de trabajo la empieza a cuestionar, se da cuenta de que desea casarse algún día, pero no está segura de la razón ni de qué quiere de un potencial esposo. Ella parece representar a las mujeres que tienen naturalizados los modelos hegemónicos de feminidad, pues los buscan seguir, pero no de forma consciente, sino automática. Es representada como una mujer gentil, buena cocinera, a quien le gustan los niños, que viste de acuerdo con la moda promovida como femenina, no tiene cualificaciones especiales y no es una mujer de carácter fuerte o dominante. Cuando se da cuenta de que tiene más de cinco años sin tener una pareja y que es incapaz de conseguir lo que otras mujeres parecen lograr de forma "natural", comienza a cuestionarse a sí misma y a intentar, con cierta desesperación, conseguir marido.

Por otro lado, Haruko, de 44 años, es una exitosa diseñadora de jardines, empleada por un corporativo especializado. Es representada como la arquetípica ohitorisama: económicamente estable e independiente, vive sola en su propio departamento, declara tener ahorros suficientes, disfruta de sus pasatiempos y de su vida sin anhelar una pareja o una familia. Y también es representada como un poco masculina al no cocinar, trasladarse en motocicleta, y vestir sólo camisetas sencillas, pantalones y botas de piso, lo cual refuerza el simbolismo de que se encuentra fuera de la feminidad hegemónica. A lo largo de la historia, Haruko declara que no siente la necesidad de casarse y que ha elegido dedicarse a su trabajo y permanecer soltera y sin hijos, remarcando que su trabajo es su compañero de vida y que, si ella se entrega a él, éste no la traicionará; constantemente señala que los jardines que diseña son sus hijos y su legado.

La serie también presenta el punto de vista de la soltería masculina. Kudo Junpei (interpretado por Tamaki Hiroshi) tiene 32 años y trabaja como empleado de tiempo parcial en una florería a la que llegará Haruko como gerente. Aunque su vocación es la pintura, renunció a ésta luego de perder un concurso contra una de sus colegas menores. Junto con el concurso, perdió la confianza en su talento y en su futuro en general, renunciando también a la idea de tener una relación sentimental y de casarse, pues cree que no está ni emocional ni económicamente preparado para ello. Por medio de estas características y al ser un pintor trabajando en una florería, es claramente representado como un sōshoku danshi.

Otra postura masculina es la del profesor Tanigawa (interpretado por Koichi Mantarō), un hombre de 46 años, divorciado y sin hijos que imparte un seminario sobre sociedad contemporánea en la universidad. Él es repre- 
sentado como poseedor de las típicas características masculinas de un hombre de mediana edad que, al verse solo por tener a su madre enferma e internada en un asilo, comienza a anhelar tener una compañera con quien compartir el día a día.

A lo largo del drama las posturas de diferentes actores sociales son expresadas por personajes secundarios. La compañera de trabajo de Chiharu representa a las mujeres japonesas que activamente tratan de encontrar un marido y desean convertirse en sengyōshufu, pero que sus demandas hacia los potenciales esposos están basadas en los ideales de la época del milagro económico, las cuales son difícilmente cumplidas por los solteros japoneses contemporáneos. Por otro lado, un compañero de 23 años personifica el punto de vista de los hombres solteros que entran dentro del modelo hegemónico de masculinidad y, conforme expresa sus opiniones sobre el matrimonio, hace que Chiharu se dé cuenta de que ella no cumple con las expectativas de una esposa de la mayoría de los hombres. Tres amigas de Chiharu y su hermana menor representan a las mujeres "ganadoras", quienes casi de forma natural se han casado y convertido en madres.

Los estudiantes del seminario de Tanigawa representan a la generación de jóvenes que pronto se convertirán oficialmente en adultos y que comienzan a darse cuenta de que cada decisión que toman afecta su futuro. El punto de vista de la generación mayor está representado por los padres de Chiharu, una típica pareja que creció durante el milagro económico y, por tanto, reproducen los discursos hegemónicos sobre el curso de vida ideal de una mujer, por lo que continuamente expresan preocupación por la soltería de su hija.

Las voces de la industria están representadas por los empleadores de los personajes principales. El hecho de que, a pesar de la demostrada capacidad de Haruko como diseñadora, sea transferida a administrar una florería, y que el contrato temporal de Chiharu no sea renovado, a pesar de sus ideas para nuevos proyectos, implica las dificultades que las solteras arafō enfrentan en el campo laboral, en donde no se les valora por su capacidad y sus habilidades, sino por su edad y su estado civil.

En la trama, Haruko y Chiharu confrontan sus visiones acerca de la soltería y el matrimonio. Al final, Haruko se mantiene muy consciente de sus elecciones y firme en su decisión de permanecer sin casarse y sin hijos, pero acepta, bajo sus propios términos, una relación con el profesor Tanigawa. Por su parte, Chiharu, a quien se le presentaron dos oportunidades de matrimonio con hombres que cumplían con los parámetros hegemónicos de masculinidad, 
se da cuenta que casarse no es lo que la hará feliz. Ella y Junpei desarrollan sentimientos el uno por el otro, pero están conscientes de que cada uno debe definir sus propias metas y forma de vida y concluyen que el matrimonio no es para ellos, por lo que no quedan como pareja.

Hay unas escenas que simbólicamente refuerzan el mensaje principal de la historia. En el primer episodio, Chiharu va a un jardín de noche a beber cerveza. Mientras está bebiendo, hace un avión de papel y lo avienta hacia una fuente que está al centro del jardín. Haruko, al ver que el avión cae en la fuente, se apresura dentro de ésta para sacarlo, temiendo que el drenaje se tape. Chiharu también entra. Un oficial de policía pasa y las lleva detenidas a la estación para interrogarlas por estar haciendo ruido de noche en un espacio público. Al final de ese episodio, Haruko y Chihahu vuelven al jardín y comienzan a gritar: “¿Qué tiene de malo no estar casada?”, “iLas mujeres no somos máquinas de hacer bebés!". El mismo oficial aparece y las lleva de nuevo a la estación de policía, pero esta vez ellas comienzan a defenderse argumentando que no han hecho nada ilegal. En el último episodio de la serie, Chiharu y Haruko están de nuevo de noche en ese jardín. Haruko le muestra a Chiharu un formato de registro de matrimonio en blanco y deciden hacer un avión de papel con él y lanzarlo. El mismo oficial de policía aparece y el avión golpea su cabeza. Ellas huyen diciendo sayonara ("adiós").

En estas escenas el policía puede verse como símbolo del seken y del gobierno. Cuando las cuestiona durante el primer episodio, se presenta una metáfora de ellas estando fuera del orden hegemónico, incluso sin estar fuera de la ley. Esto representa cómo el seken ve a las arafō solteras como si se opusieran al "orden natural" y cómo cuestiona su comportamiento. Luego de que Haruko y Chiharu, a lo largo de su proceso de introspección durante la historia, refuerzan su postura respecto a seguir solteras, su lanzamiento del formato de registro de matrimonio hecho avión de papel y golpeando la cabeza del oficial simboliza la resistencia que ellas están poniendo ante la idea hegemónica del seken de que el matrimonio debe ser la meta de todas las mujeres. La escena representa un reclamo a su libertad para decidir por sí mismas lo que las haga sentirse satisfechas con sus vidas.

Por otro lado, Watashi kekkon dekinainjanakute, shinaindesu está basado en Suparuta konkatsujuku (Mizuno, 2014), un manual sobre cómo conseguir marido. La historia fue adaptada por la guionista Kaneko Arisa — una arafōpara crear una historia enfocada en la vida de Tachibana Miyabi (interpretada por Nakatani Miki), una dermatóloga de 39 años que encaja perfectamente 
con el arquetipo de ohitorisama. Es dueña de una clínica estética y un grande y elegante departamento ubicados en una de las zonas de clase alta en Tokio; se preocupa mucho por su salud y belleza, pero no sabe cocinar; tiene un estilo de vestir sofisticado y declara que odia el estilo de presentadora de televisión - refiriéndose a la moda femenina dominante-; disfruta leer historietas con temas para chicos; vive sola con su perro; $y$ no ha tenido pareja por cinco años.

Miyabi siempre ha sido reconocida por la gente a su alrededor como una mujer hermosa, inteligente y profesionalmente exitosa. Sin embargo, durante una reunión con sus compañeros de preparatoria - al igual que Satoko en Around 40 - se ve de pronto enfrentando miradas de lástima y recibiendo palabras de ánimo cuando sus compañeros se enteran de que no está casada ni tiene novio. Al darse cuenta de que ellos piensan que esto es debido a que no ha podido conseguir a alguien, ella declara que no es que no pueda casarse, sino que no lo ha hecho debido a que se ha enfocado en cosas más relevantes para ella. El mismo día, durante una cena con dos amigas también arafō solteras, es confrontada por el dueño y chef del restaurante, Tokura Seiji (interpretado por Fujiki Naohito) de 42 años. Él le dice que, para el seken, las arafō que son solteras, bellas y orientadas a sus carreras son consideradas makeinu, pues esas características son vistas como deméritos por los varones, volviéndolas incapaces de encontrar un esposo. Miyabe acude a un servicio de matrimonios arreglados y, una vez más, le dicen que el ser una arafō con una carrera especializada le daba pocas posibilidades de encontrar un esposo, pues la mayoría de los hombres preferían a mujeres de menos de 35 años y que tuvieran sólo empleos temporales.

Esos encuentros con la visión del seken de ella como perdedora, comienzan a hacerla dudar de su estatus de soltera y, cuando se reencuentra por casualidad con Sakurai Yosuke (interpretado por Tokui Yoshimi), un compañero de la preparatoria que fue su amor no correspondido de adolescencia, se propone conquistarlo. Yosuke es representado como un salaryman con una carrera bien establecida y experiencia viviendo en el extranjero, por lo que es popular con las mujeres. El drama presenta un ciclo completo en la postura de Miyabi respecto al matrimonio. Al inicio, confiada en sí misma, podía declarar firmemente que su soltería era por elección. No obstante, al notar que el seken la consideraba una perdedora, etiqueta a la cual ella no estaba acostumbrada, comienza un viaje simbólico en pos de encontrar una pareja. Sin embargo, en esa búsqueda se pierde a sí misma en las expectativas y deseos de la sociedad, particularmente de los varones. Luego de muchas 
tácticas y adecuaciones que Miyabi hace de sí misma y de sus expectativas hacia una pareja, Yosuke le propone matrimonio. Entonces, se da cuenta que casarse no era algo que deseara y que, al intentar ser exitosa en los términos del seken, había sacrificado su satisfacción y visión de sí misma. Al final del drama, Miyabi deja a Yosuke y va a decirle a Tokura sus conclusiones: "No es que no me pueda casar, es que no me caso".

\section{Reflexiones finales}

Durante las últimas décadas Japón ha sufrido profundos cambios sociales que han impactado la conciencia de las personas en términos de su identidad de género y trayectorias de vida. En ese contexto, los discursos hegemónicos han puesto a las personas solteras de más de 30 años como personificaciones de los quiebres entre el próspero pasado, el difícil presente y el incierto futuro, por no adecuarse a los deseos del seken y a los objetivos nacionales. Esto se ha visto reflejado en los estereotipos que circulan sobre ellas y sus elecciones de vida, y en el uso de etiquetas que afectan cómo son vistas por la sociedad y cómo se perciben ellas mismas. No obstante, a través de los medios comienza a percibirse una incipiente resistencia.

En Around 40, Kekkon shinai y Watashi... el discurso dominante es que las mujeres que se encuentran fuera de la feminidad hegemónica tienen dificultades para encontrar una pareja que las comprenda y acepte tal como son, reforzando la idea de que, a nivel social, el matrimonio es considerado como el máximo símbolo del éxito y la felicidad para la mujer. No obstante, hay una evolución en la resolución de las historias. Las protagonistas se prueban a ellas mismas que pueden conseguir lo que el resto de las mujeres parecen obtener naturalmente, pero que ni el matrimonio ni la maternidad son para ellas, revalorizando sus logros, recuperando el control sobre su propia construcción identitaria y rechazando finalmente la presión externa.

Por otro lado, tanto en Kekkon Dekinai Otoko como en el resto de las series analizadas, en el caso de los varones solteros se les percibe como extraños al no estar casados, pero cumplen con la esencia de masculinidad hegemónica al tener éxito laboral, el cual es socialmente reconocido y aceptado como fuente de felicidad para ellos. Aquellos que aún no tienen éxito laboral, por su parte, son representados como fuera de la masculinidad hegemónica, pero son reivindicados al arriesgarse a buscar ese éxito, a costa incluso de la estabilidad económica. 
Butler (1990) considera que la cuestión de la agencia descansa en si las normas son consolidadas o resignificadas a través de su citación o su repetición continua. Luego del análisis hecho de estas series, se pueden observar pequeños espacios de resignificación en donde algunas personas solteras están incrementando su nivel de agencia al elegir no repetir los papeles de género y trayectorias de vida tradicionales, a pesar de la presión social y gubernamental.

Ante la mayor diseminación de contenidos audiovisuales a través ya no sólo de televisión tradicional, sino también de sitios streaming, se pueden incrementar las oportunidades para reconstruir el discurso dominante alrededor de la soltería y el rechazo a la maternidad/paternidad por parte de los adultos japoneses que así se identifican. Desde la producción, se presentan cada vez más espacios para la realización creativa; y desde el consumo, ante la diversidad de oferta, se puede presionar más a las élites para que cambien los discursos que resultan limitantes u ofensivos.

\section{Referencias}

Buckley, S. (1993). Altered states: the body politics of "being-woman". En A. Gordon (Ed.), Postwar Japan as History (pp. 347-372). University of California Press.

Butler, J. (1990). Gender Trouble: Feminism and the Subversion of Identity. Routledge.

Cox, P. (2015, septiembre 09). Matahara: When pregnant women, new moms are harassed at work. The World. http://www.pri.org/stories/2015-09-09/ maternity-harassment-so-common-japanese-workplace-they-inventedword-it

Dales, L. (2014). Ohitorisama, Singlehood and Agency in Japan. Asian Studies Review, 38(2), 224-242. https://doi.org/10.1080/10357823.2014.9020 33

Dasgupta, R. (2010). Globalisation and the Bodily Performance of 'Cool' and 'Un-cool' Masculinities in Corporate Japan. Intersections: Gender and Sexuality in Asia and the Pacific, (23). http://intersections.anu.edu. au/issue23/dasgupta.htm

Fujimura-Fanselow, K. (1991). The Japanese Ideology of 'Good Wives and Wise Mothers': Trends in Contemporary Research. Gender \& History, 3(3), 345-349. https://doi.org/10.1111/j.1468-0424.1991.tb00136.x 
Hidaka, T. (2010). Salaryman Masculinity: The Continuity of and Change in the Hegemonic Masculinity in Japan: Vol. 29. Social Sciences in Asia. Brill. https://doi.org/10.1163/ej.9789004183032.i-224

Ishida, H. (2013). The Transition to Adulthood among Japanese Youths: Understanding Courtship in Japan. The Annals of the American Academy of Political and Social Science, 646(1), 86-106. https://doi. org/10.1177/0002716212465589

Ivy, M. (1993). Formations of Mass culture. En A. Gordon (Ed.), Postwar Japan as History (pp. 239-258). University of California Press.

Iwashita, K. (2001). Ohitorisama [Una persona]. Chuokoronshinsha.

Lebra, T. S. (2004). The Japanese Self in Cultural Logic. University of Hawaii Press.

Luckács, G. (2010). Scripted Affects, Branded Selves: Television, Subjectivity, and Capitalism in 1990s Japan. Duke University Press.

Mandujano-Salazar, Y. Y. (2017). It is Not that I Can't, It is that I Won't: The Struggle of Japanese Women to Redefine Female Singlehood through Television Dramas. Asian Studies Review, 41(4), 526-543. https://doi.or $\mathrm{g} / 10.1080 / 10357823.2017 .1371113$

McKinlay, M. (2002). Unstable Mothers: Redefining Motherhood in Contemporary Japan. Intersections: Gender, History and Culture in the Asian Context, (7). http://intersections.anu.edu.au/issue7/mckinlay.html

Mizuno, K. (2014). Suparuta konkatsujuku [Escuela espartana para conseguir pareja para matrimonio]. Bunkyosha.

National Institute of Population and Social Security Research. (s.f.). Selected Demographic Indicators for Japan. National Institute of Population and Social Security Research Web Page. http://www.ipss.go.jp/p-info/e/S_D_I/ Indip.asp

National Institute of Population and Social Security Research. (2017). Population Statistics of Japan 2017. National Institute of Population and Social Security Research Web Page. http://www.ipss.go.jp/p-info/e/psj2017/ PSJ2017.asp

Niwa, A., \& Yoda, T. (1993). The Formation of the Myth of Motherhood in Japan. U.S.-Japan Women's Journal. English Supplement, (4), 70-82. http:// www.jstor.org/stable/42772053

Prime Minister of Japan and His Cabinet. (2014, marzo 28). Council for Supporting Women to Shine. http://japan.kantei.go.jp/96_abe/ actions/201403/28woman.html 
Rosenberger, N. (2007). Rethinking Emerging Adulthood in Japan : Perspectives From Long-Term Single Women. Child Development Perspectives, 1(2), 92-95. https://doi.org/10.1111/j.1750-8606.2007.00021.x

Ryan, K. (2015, febrero 11). Maternity Harassment in Japan. The Diplomat. http://thediplomat.com/2015/02/maternity-harassment-in-japan/

Sakai, J. (2003). Makeinu no tōboe [El aullido de los perros perdedores]. Kodansha.

Takemaru, N. (2010). Women in the Language and Society of Japan: The Linguistic Roots of Bias. McFarland \& Company.

The Government of Japan. (2018). ABENOMICS. https://www.japan.go.jp/ abenomics/index.html

Uno, K. S. (1993). The death of "Good wife, wise mother"? En A. Gordon (Ed.), Postwar Japan as History (pp. 293-323). University of California Press.

Upham, F. K. (1993). Unplaced Persons and Movements for Place. En A. Gordon (Ed.), Postwar Japan as History (pp. 325-346). University of California Press.

Video Research Ltd. (s.f.). Video Research. https://www.videor.co.jp/

Vogel, E. (1971). Japan's New Middle Class: The Salary Man and His Family in a Tokyo Suburb (Segunda edición). University of California Press. https:// doi.org/10.1177/089124167300200312

Yamada, M. (1999). Parasaito Shinguru no Jidai [La era de los solteros parásito]. Chikuma Shobo.

Yu, W.-H. (2009). Gendered Trajectories. Women, Work, and Social Change in Japan and Taiwan. Stanford University Press. 